\title{
Loss of miR-449a-caused PrLZ overexpression promotes prostate cancer metastasis
}

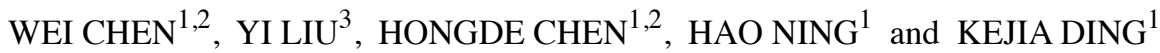 \\ ${ }^{1}$ Department of Urology, Shandong Provincial Hospital Affiliated to Shandong University, Jinan, Shandong 250021; \\ ${ }^{2}$ Department of Urology, The First Affiliated Hospital of Wenzhou Medical University, Wenzhou, \\ Zhejiang 325000; ${ }^{3}$ Department of Gynaecology and Obstetrics, The Second Affiliated Hospital \\ of Wenzhou Medical University, Wenzhou, Zhejiang 325000, P.R. China
}

Received March 23, 2017; Accepted May 24, 2017

DOI: 10.3892/ijo.2017.4038

\begin{abstract}
Distant metastasis is the worst prognostic factor for PCa patients. It has been reported that miR-449a enhances radiosensitivity of prostate cancer cells, but the function of miR449a in metastasis of prostate cancer is mainly unknown. In the present study, we strove to investigate the function and diagnostic value of miR-449a in metastasis of prostate cancer. qRT-PCR was used to quantify the expression of miR449a and PrLZ in PCa cell lines and tissues. We found that miR449a expression was decreased in PCa cell lines. Moreover, miR-449a was downregulated in PCa tissues, especially in primary lesion tissues of metastatic PCa patients. CCK8, FACS, Transwell and tube formation assay were performed to assess growth and metastasis of PCa cells in vitro. Lentivirus mediated miR-449a overexpression suppressed proliferation of LNcap and PC-3, and miR-449a also significantly inhibited invasion and angiogenesis ability of LNcap and PC-3. IHC showed that PrLZ was upregulated in PCa tissues. Luciferase assay and western blotting verified that miR-449a targeted PrLZ expression. Moreover, PrLZ shRNA also significantly suppressed proliferation and metastasis of LNcap and PC-3. In addition, western blotting revealed that miR-449a overexpression and PrLZ shRNA all remarkably inhibited the stemness features in LNcap and PC-3. Furthermore, BALB/c nude mouse subcutaneous xenograft model was uesd to verify the function of miR-449a and PrLZ. Our results showed that miR-449a and PrLZ shRNA significantly suppressed PC-3 tumorigenesis and metastasis in vivo. Our studies suggested that miR-449a decreased in malignant process of PCa and was accompanied by excess expression of PrLZ. The loss of
\end{abstract}

Correspondence to: Professor Kejia Ding, Department of Urology, Shandong Provincial Hospital Affiliated to Shandong University, Jinan, Shandong 250021, P.R. China

E-mail: dingkejia_shandong@126.com

Abbreviation: PCa, prostate cancer; PI, propidium iodide; UTR, untranslated region; LV-miR-449a OE, miR-449a overexpression lentivirus; LV-PrLZ shRNA, PrLZ shRNA lentivirus

Key words: miR-449a, PrLZ, prostate cancer, metastasis
miR-449a caused PrLZ overexpression regulated prostate cancer progression and metastasis via regulating the stemness features of prostate cancer cells. The diagnostic value of miR-449a as a distant metastasis predictor of PCa needs further investigation.

\section{Introduction}

Prostate cancer ( $\mathrm{PCa}$ ) is one of the most common malignancies in males in many countries (1). According to statistics in 2016, there were approximately 3 million men suffering from prostate cancer in the United States, and additionally, more than 180,000 new cases were reported (2). In the past decades, the incidence and mortality of PCa also significantly increased in China (3). Studies have confirmed that distant metastasis is the major cause of PCa-related mortality $(4,5)$.

At present, PSA level is used as an index for predicting PCa progression include bone metastasis in Western countries $(6,7)$. However, there were studies considered it could be different in Asian patients, researchers found bone metastatic PCa patients in Asian with lower PSA level (7). It is necessary to exploit new predictive factors and diagnosis patterns for PCa distant metastasis.

MicroRNAs are a class of non-coding small RNAs, acting as negative regulators of gene expression through direct interaction with the 3'-untranslated regions (3'-UTR) of corresponding mRNA targets (8). Previous studies had extensively confirmed that MicroRNAs played vital roles in tumor metastasis (9-11). Nevertheless, there are very few comprehensive reports on the prediction and diagnosis values of microRNAs in metastatic PCa (12).

PrLZ was identified as a novel member of the TPD52 family which is specifically expressed in prostate tissues (13). Previous studies proved that PrLZ was associated with the progression of prostate cancer (14). Increased PrLZ promoted prostate cancer growth at castration-resistant stage and protected prostate cancer cells from apoptosis induced by androgen deprivation (15). However, the regulation mechanism and function of PrLZ in PCa distant metastasis is unclear.

In our previous study, we identified that miR-449a was significantly decreased in primary lesion tissues of bone metastatic PCa patients. In this study, we further investigated the 
Table I. Demographic information of patient.

\begin{tabular}{lccc}
\hline Patient charectaristics & Distant metastasis & No distant metastasis & Total \\
\hline Number & 17 & 21 & 38 \\
Age (years) & Age range, $42-74$ (mean 60.18) & Age range, $41-70$ (mean 55.9) & \\
Stage & & & 19 \\
I-II & 4 & 6 & 19 \\
III-IV & 13 & $41.8 \pm 19.81$ & \\
PSA $(\mathrm{ng} / \mathrm{ml})$ & $22.5 \pm 9.59$ & \\
\hline
\end{tabular}

function of miR-449a in the process of prostate cancer metastasis. Furthermore, we demonstrated that miR-449a inhibited prostate cancer metastasis via targeting PrLZ expression.

\section{Materials and methods}

Patients and specimens. Prostate cancer tissues were collected from patients who underwent curative resection with informed consent between 2011 and 2014 at the Department of Urinary Surgery, the First Affiliated Hospital of Wenzhou Medical University. These tissues were immediately transported into liquid nitrogen in operating theatres and then stored at $-80^{\circ} \mathrm{C}$. In the present study, specimens were divided into two groups according to whether the patients relapsed with distant metastasis in two years after surgical operation. The demographic information of patients is shown in Table I. The study protocol was approved by the Ethics Committee of the First Affiliated Hospital of Wenzhou Medical University, and written informed consents were obtained from the patients based on the Declaration of Helsinki.

Cell culture. The human normal prostate epithelial cell line RWPE-1 and PCa cell lines PC-3, LNcap cells were purchased from the American Type Culture Collection (ATCC; Manassas, VA, USA). PC-3 and LNcap cells were cultured in RPMI-1640 medium with $10 \%$ fetal bovine serum (FBS; Life Technologies, Foster City, CA, USA), 100 units/ml penicillin and 100 units/ml streptomycin (Sigma-Aldrich, St. Louis, MO, USA). RWPE-1 cells were cultured in Defined Keratinocyte-SFM (Life Technologies, Carlsbad, CA, USA) with $10 \% \mathrm{FBS}, 100$ units $/ \mathrm{ml}$ penicillin and 100 units $/ \mathrm{ml}$ streptomycin. All these cells were cultured in a humidified environment containing $5 \% \mathrm{CO}_{2}$ and held at a constant temperature of $37^{\circ} \mathrm{C}$.

qRT-PCR. Total RNAs for PrLZ and $\beta$-actin were extracted from clinical samples using TRIzol reagent (Life Technologies). cDNA was synthesized using the PrimeScript RT reagent kit (Takara Bio, Mountain View, CA, USA). The primers used for the expression analysis of $\beta$-actin and PrLZ were as follow: $\beta$-actin forward, 5'-GGCACTCTTCCAGCCTTCC-3' and $\beta$-actin reverse, 5'-GAGCCGCCGATCCACAC-3'; PrLZ forward, 5'-TCTAGCAGAGATCAAGCGGAA-3' and PrLZ reverse, 5'-ACTGAGCCAACAGACGAAAAA-3'. Total RNAs for miR-449a and U6 were extracted from clinical samples and cells using miRNeasy Mini kit (Qiagen, Hilden, Germany). The qRT-PCR reactions of miR-449a and U6 were performed according to the manufacturer's instructions of All-in-One ${ }^{\mathrm{TM}}$ miRNA qRT-PCR detection kit (GeneCopoeia, Rockville, MD, USA). iQ-5 (Bio-Rad Laboratories, Hercules, CA, USA) was used to monitor the qRT-PCR reactions. RNA expression was quantified using $2^{-\Delta \Delta C t}$ method.

Western blotting. Total protein was extracted using RIPA protein lysis buffer (Beyotime Institute of Biotechnology, Shanghai, China) with added $1 \%$ protease inhibitor cocktail and $1 \mathrm{mM}$ phenylmethylsulfonyl fluoride (PMSF). Cell fractions were prepared using a Nuclear and Cytoplasmic Protein Extraction kit (Beyotime Institute of Biotechnology) according to the manufacturer's protocol. Generally, $50 \mu \mathrm{g}$ of protein was used for western blotting. Samples were separated by SDS-PAGE and transferred onto PVDF membranes. After blocking in 5\% skim milk, the PVDF membranes were incubated with primary antibodies in blocking buffer overnight at $4^{\circ} \mathrm{C}$ and then with HRP-conjugated secondary antibody for $2 \mathrm{~h}$. The primary antibodies used were: anti-GAPDH (1:3,000 dilution; Santa Cruz Biotechnology, Santa Cruz, CA, USA), anti-PrLZ (1:1,000 dilution; Abcam, Cambridge, UK), anti-E-cadherin (1:1,000 dilution; Abcam), anti-N-cadherin (1:1,000 dilution; Abcam), anti-CD44 (1:1,000 dilution; Abcam), anti-vimentin (1:1,000 dilution; Abcam), anti-MMP2 (1:1000 dilution; Abcam), anti-MMP9 (1:500 dilution; Abcam), anti-RunX2 (1:500 dilution; Abcam). Reactive bands were visualized with ECL reagent (Pierce, Rockford, IL, USA) and analyzed. Protein expression was quantified using ImageJ software (National Institutes of Health, Bethesda, MD, USA).

Immunohistochemistry (IHC). Formalin-fixed, paraffinembedded tissues were used to detect the expression of PrLZ and $\mathrm{N}$-cadherin. The sections were incubated with anti-PrLZ and anti-N-cadherin rabbit polyclonal antibodies (Abcam) at 1:200 dilution. A semi-quantitative scoring system was used to evaluate the intensity of staining: low (proportion, $0-50 \%$; intensity, no staining to weak) and high (proportion, $>50 \%$; intensity, intermediate to strong).

Oligonucleotide transfection and luciferase reporter assay. miR-146a mimics and scramble control mimics were purchased from GeneCopoeia Inc. (Rockville, MD, USA). PC-3 cells $\left(10^{5}\right.$ cells) were seeded in 24 -well plates before transfection. Wild-type PrLZ 3'UTR (PrLZ-3'UTR-wt) and miR449a target site deletion mutation PrLZ 3'UTR (PrLZ-3'UTR-mu) were constructed into psiCHECK2 plasmid (Promega, Madison, WI, USA). According to the instructions, miR-449a mimic and 

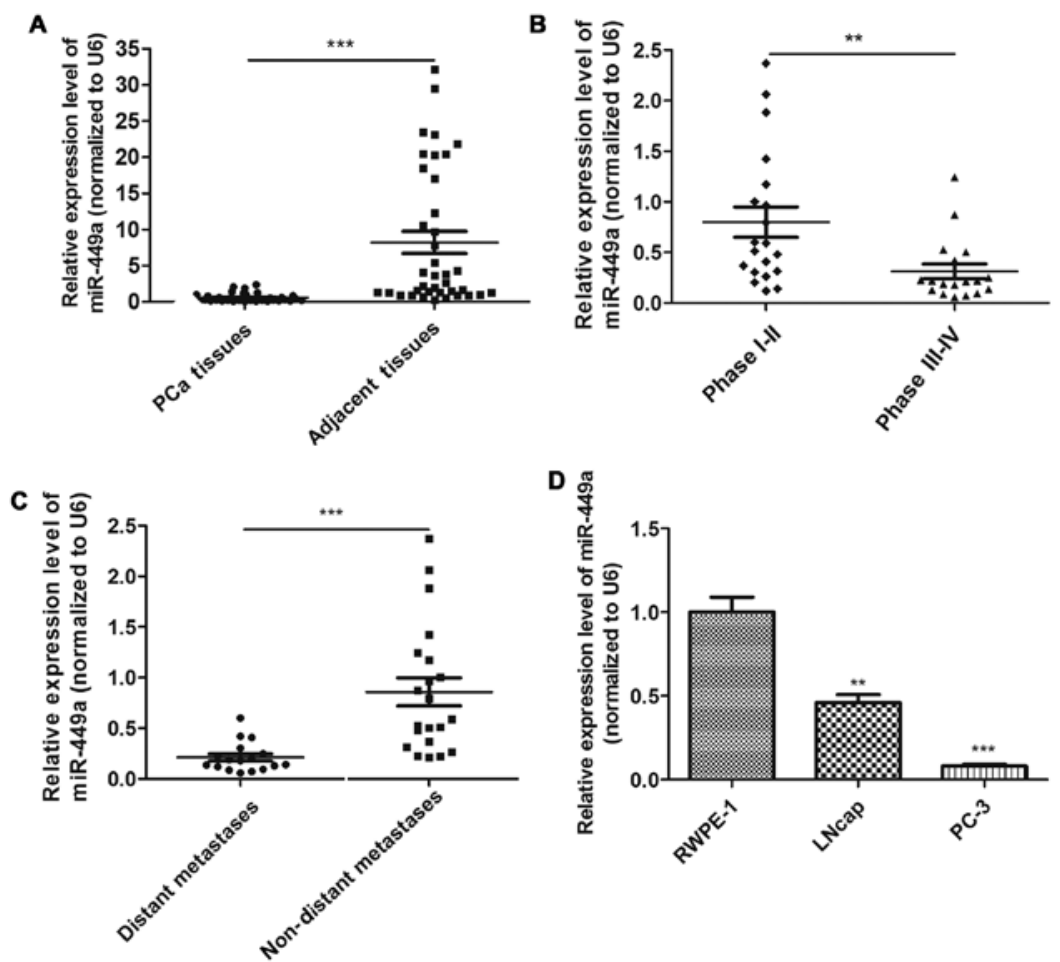

Figure 1. miR-449a is downregulated in PCa tissues and cell lines. (A) The expression of miR-449a was detected in PCa tissues and adjacent tissues using qRT-PCR. miR-449a was significantly downregulated in PCa tissues. ${ }^{* * *} \mathrm{P}<0.001$, paired two-tailed Student's t-test. (B) miR-449a expression level was remarkably lower in phage III-IV PCa patients compared with phage I-II. ${ }^{* *} \mathrm{P}<0.01$, unpaired two-tailed Student's t-test. (C) The expression of miR-449a was maintained at a lower level in PCa patients who were recurrent with bone metastasis in two years after surgical operation. ${ }^{* *} \mathrm{P}<0.01$, unpaired two-tailed Student's t-test. (D) The expression of miR-449a was detected in PCa cells and RWPE-1 using qRT-PCR. miR-449a was decreased in PCa cell lines. ${ }^{* *} \mathrm{P}<0.01$, ${ }^{* * *} \mathrm{P}<0.001$, unpaired two-tailed Student's t-test.

PrLZ 3'UTR Reporter plasmids (psiCHECK-PrLZ-3'UTR-wt or psiCHECK-PrLZ-3'UTR-mu) were co-transfected using Lipofectamine ${ }^{\circledR}$ RNAiMAX (Life Technologies) with final concentration of $50 \mathrm{nM}$ (miR-146a mimic) or $200 \mathrm{ng}$ (PrLZ 3'UTR Reporters). In addition, $48 \mathrm{~h}$ later, the cells were collected and Luciferase activity was detected using the Dual-Glo luciferase assay system (Promega) according to the manufacturer's protocol.

Lentivirus infection. miR-449a overexpression and PrLZ shRNA lentivirus were purchased from Shanghai R\&S Biotechnology, Co., Ltd. (Shanghai, China). Cells were planted into $10-\mathrm{cm}$ dishes $\left(3 \times 10^{6}\right.$ cells/dish) $24 \mathrm{~h}$ before the infection. Lentivirus infection was performed with the MOI (multiplicity of infection) of 30 (LNcap) and 50 (PC-3), respectively. The infection efficiency was $>90 \%$.

Cell proliferation analysis. Cells were seeded in 96-well plates in triplicate at densities of $5 \times 10^{3} /$ well. Cell viability was evaluated at desired time-points using CCK- 8 kits (Dojindo Molecular Technologies, Kumamoto, Japan) according to the instructions. Light absorbance of the solution was measured at $570 \mathrm{~nm}$ on a microplate reader.

Cell invasion assay. Transwell chambers coated with Matrigel (BD Biosciences, San Diego, CA, USA) were used to analysis cell invasion. Du145 cells $\left(5 \times 10^{5}\right)$ in $100 \mu 1$ serum-free Dulbecco's modified Eagle's medium (DMEM) were seeded on upper chambers and DMEM with 10\% FBS was added to lower chambers. After 24-h incubation, invaded cells in the lower side of the membranes were fixed with methanol and stained with crystal violet. Images were taken using an inverted microscope. Invaded cells were counted from three different fields. The experiment was repeated three times.

Tube formation assay. Forty-eight hours after lentivirus infected, PCa cells were washed with phosphate-buffered saline (PBS) twice, and then cultured in medium containing $1 \%$ serum for another $24 \mathrm{~h}$. Conditioned media were then collected, centrifuged at $1,500 \mathrm{rpm} / \mathrm{min}$ and the supernatant was used for tube formation experiments. HUVEC $\left(2 \times 10^{4}\right.$ cells/well) were seeded onto the 96-well plates which were pre-coated with polymerized Matrigel (BD Biosciences) overnight, and then incubated with $100 \mu \mathrm{l}$ conditioned media from $\mathrm{PCa}$ cells at $37^{\circ} \mathrm{C}$ for $4 \mathrm{~h}$ in a $5 \% \mathrm{CO}_{2}$ incubator. Six random fields from each well were selected to count the branch points of tube-like structures (16).

Apoptosis assay. Cell apoptosis was determined by flow cytometry using the Annexin V-FITC apoptosis detection kit (Nanjing KeyGen Biotech, Co., Ltd., Nanjing, China). Cells were collected and incubated with Annexin V and propidium iodide (PI) in a binding buffer in the dark at room temperature for $10 \mathrm{~min}$. The stained cells were analyzed using the BD FASAria Cell Sorter.

Tumorigenesis in nude mice. Six-week-old female BALB/c nude mice were injected with PC-3 cell subcutaneously 

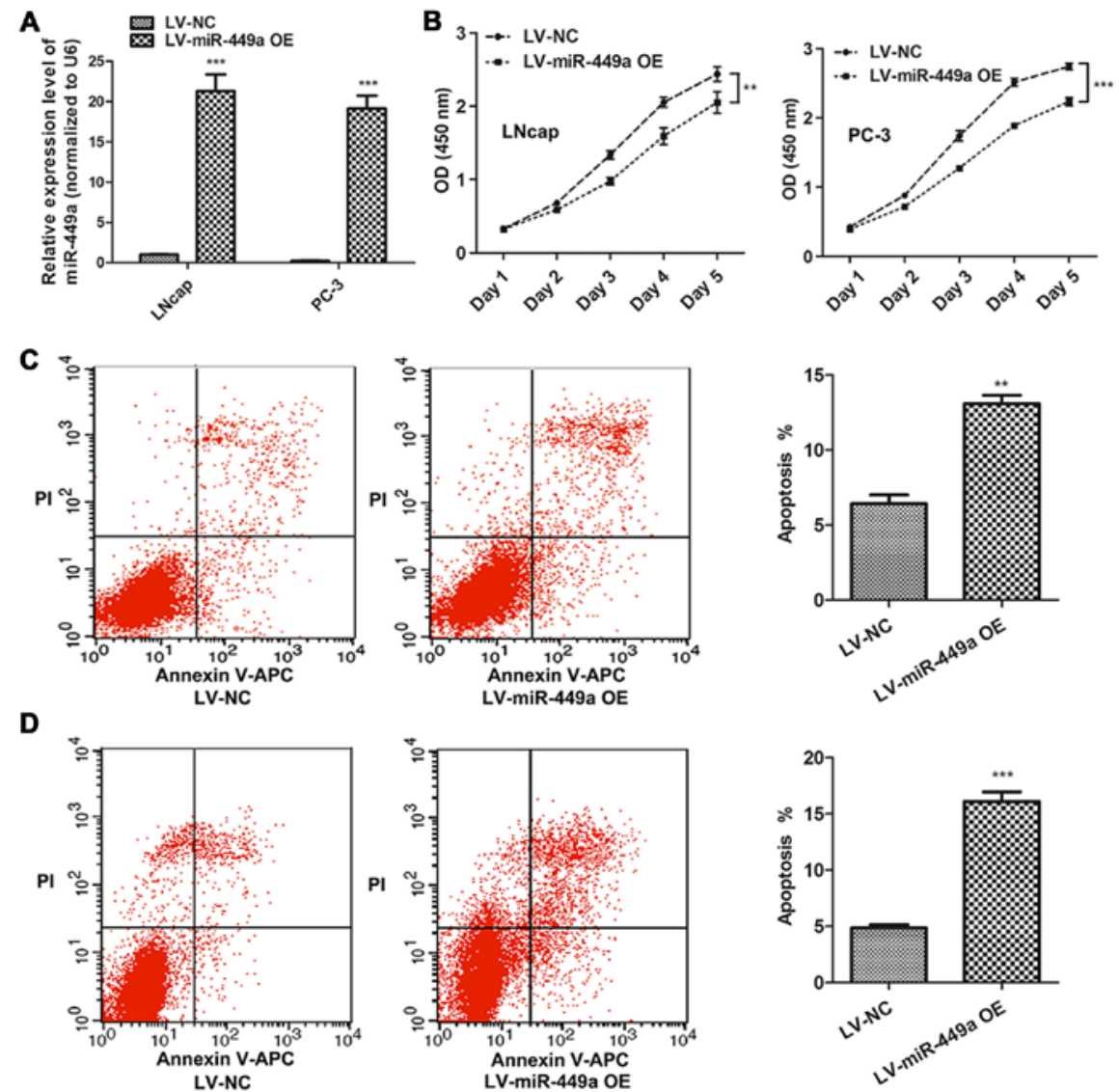

Figure 2. miR-449a suppresses proliferation and promotes apoptosis in LNcap and PC-3 cells. (A) The expression of miR-449a was detected in LNcap and PC-3 cells after miR-449a overexpression lentivirus or NC lentivirus infected by qRT-PCR. The result showed that miR-449a remarkably increased in LNcap and PC-3. ${ }^{* * *} \mathrm{P}<0.001$, unpaired two- tailed Student's t-test. (B) miR-449a overexpression delayed LNcap and PC-3 proliferation. ${ }^{* *} \mathrm{P}<0.01,{ }^{* * * *} \mathrm{P}<0.001$, one-way ANOVA. (C) miR-449a promoted LNcap apoptosis. ${ }^{* *} \mathrm{P}<0.01$, unpaired two-tailed Student's t-test. (D) miR-449a promoted PC-3 cells apoptosis. ${ }^{* * *} \mathrm{P}<0.001$, unpaired two-tailed Student's t-test.

$\left(10^{7}\right.$ cells/mouse) into the left axilla. For lentivirus infection groups, $10 \mu 1$ lentivirus were respectively injected into tumors every three days when the tumor volume up to $50 \mathrm{~mm}^{3}$. The tumor sizes were measured every three days using micrometer calipers, and tumor volumes were calculated as following: Tumor volume $=\mathrm{d}^{2} \mathrm{xD} / 2$, where $\mathrm{d}$ and $\mathrm{D}$ represented shortest and the longest diameters, respectively. All animal experiment protocols were approved by the Institutional Animal Care and Use Committee.

Statistical analysis. Results are presented as means \pm standard deviations (SD) of three independent experiments. Significant differences in the mean values were evaluated by unpaired t-test. One-way ANOVA was used to compare continuous variables among two or more groups. Tests of association were conducted using Pearson's $\chi^{2}$ test. $\mathrm{P}<0.05$ was considered statistically significant. Literature collection was performed by using electronic databases PubMed. All statistical analyses were executed by using SPSS 20.0 software (IBM, Chicago, IL, USA). Raw and processed data are stored by the corresponding author and are available upon request.

\section{Results}

Downregulation of miR-449a is related to PCa clinical stage and distant metastasis. In order to investigate whether
miR-449a was associated with PCa progression, we examined the expression of miR-449a in 38 PCa tissues using qRT-PCR. We confirmed that miR-449a was significantly downregulated in PCa tissues compared to adjacent tissues (Fig. 1A). To investigate the clinical significance of miR-449a, we assessed the correlation between miR-449a expression level and pathological characteristics. The result showed that miR-449a was remarkably decreased in stage III-IV patients compared with stage I-II patients (Fig. 1B). As previously described, PCa tissues were divided into two groups. We found that miR-449a was obviously lower expressed in distant metastasis positive specimens (Fig. 1C). miR-449a expression levels in various prostate derived cells are presented, miR-449a was remarkably downregulated in prostate cancer cell lines, especially in androgen-independent prostate cancer cell PC-3 (Fig. 1D). These results indicate that $\mathrm{miR}-449 \mathrm{a}$ is related to malignance of prostate cancer.

miR-449a inhibits PCa progression. LNcap and PC-3, which represented androgen-dependent and androgen-independent PCa cells, respectively, were used to investigate the function of miR-449a in PCa. miR-449a overexpression lentivirus (LV-miR-449a OE) was performed to upregulate miR-449a expression in PCa cells. We found that miR-449a suppressed cell proliferation in LNcap and PC-3 (Fig. 2A). In addition, flow cytometry assay showed that miR449a remarkably promoted 

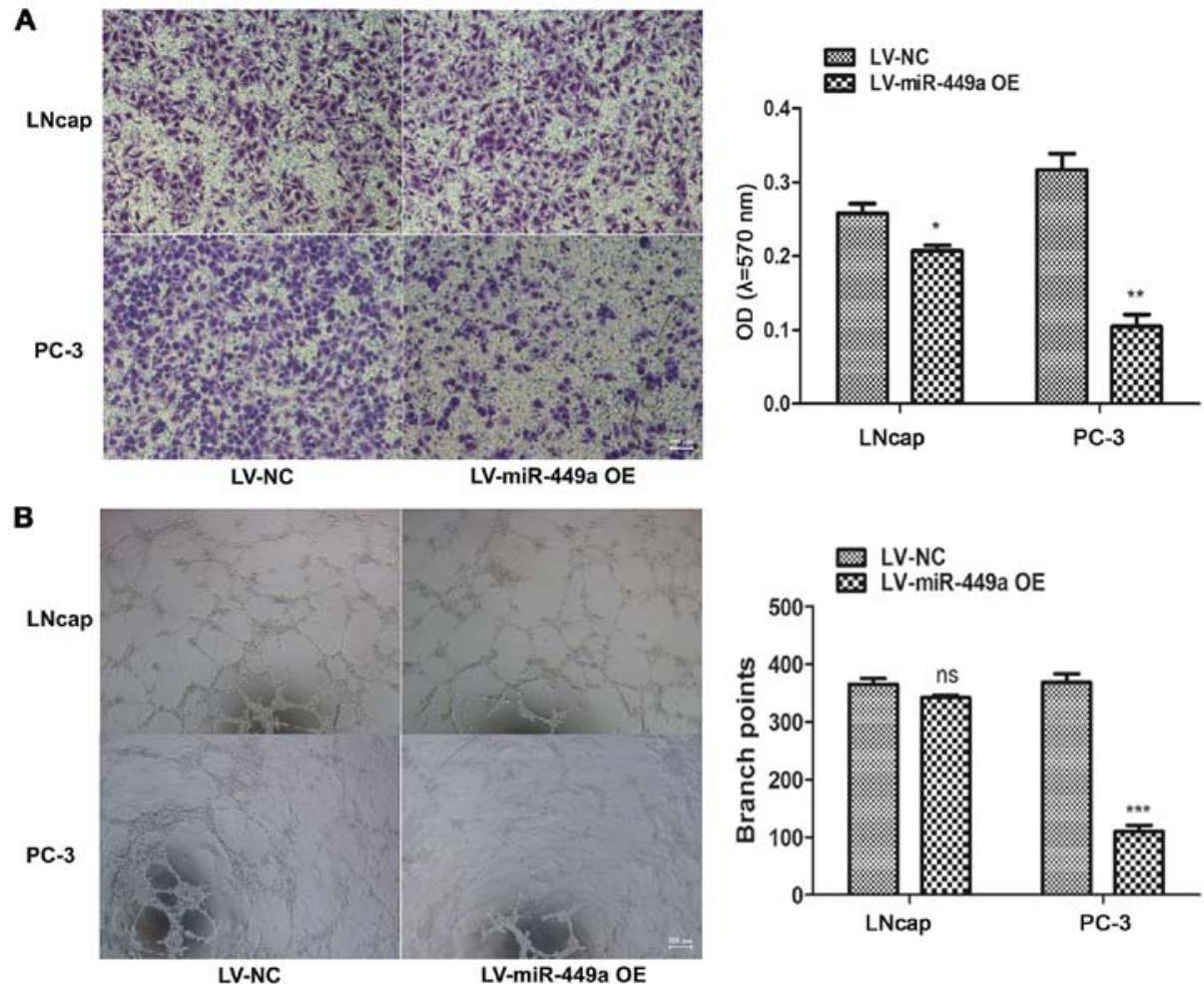

Figure 3. miR-449a inhibits invasion and angiogenesis ability in LNcap and PC-3 cells. (A) miR-449a suppressed invasion in LNcap and PC-3. "P<0.05, ${ }^{* *} \mathrm{P}<0.01$, unpaired two-tailed Student's t-test. (B) miR-449a inhibited LNcap and PC-3 induced huvec cells tube formation. ${ }^{* * *} \mathrm{P}<0.001$, unpaired two-tailed Student's t-test.

A

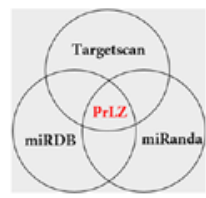

PrZZ, UTR $s$ CUUUGUUCUGCUACCCACUGCCA

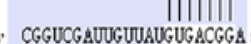

B

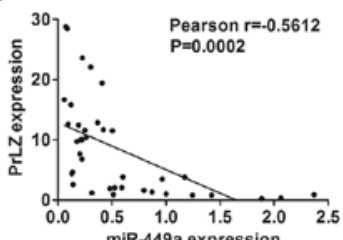

C

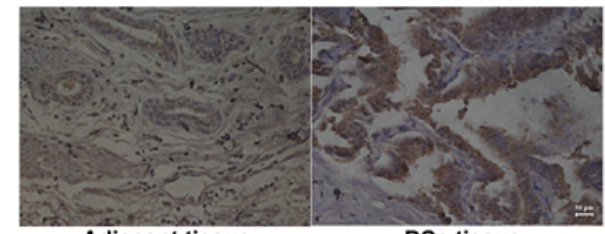

Adjacent tissue

PCa tissue

$\mathbf{E}$

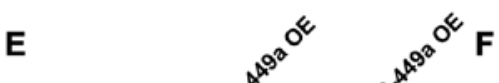

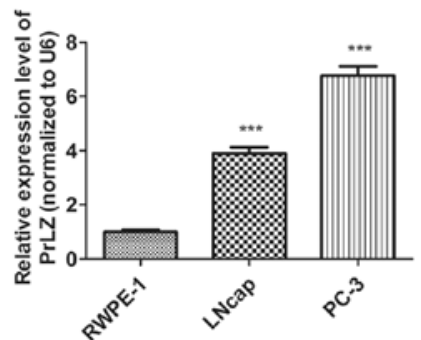
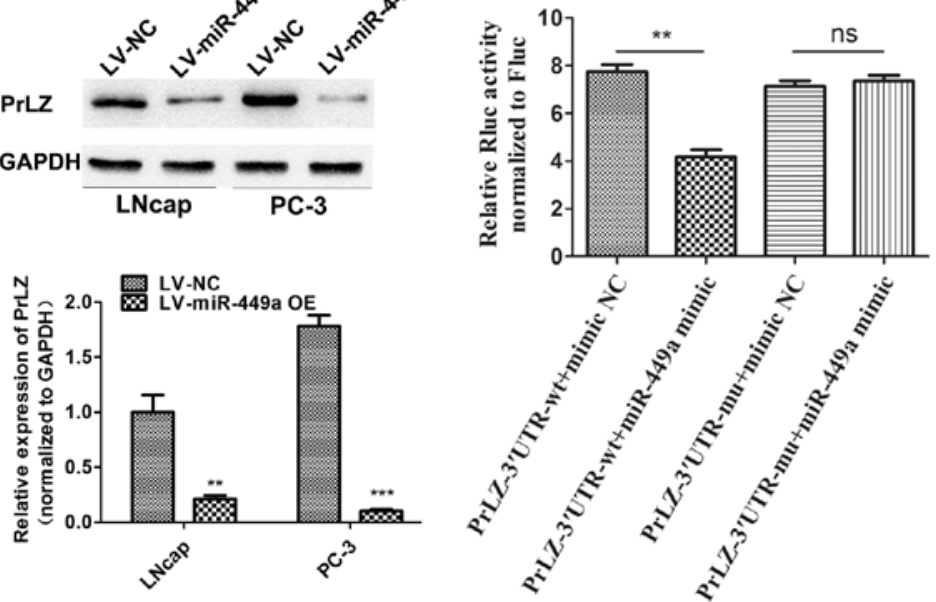

Figure 4. miR-449a targets PrLZ in PCa. (A) The target site of miR-449a in PrLZ 3'-UTR predicted combinationally by TargetScan, miRDB and miRanda. (B) Correlation analysis of miR-449a and PrLZ expression in $38 \mathrm{PCa}$ specimens. $\mathrm{P}=0.002$. (C) IHC analysis of PrLZ expression. Compared to adjacent tussues, PrLZ was significantly upregulated in corresponding PCa tissues. (D) The expression of PrLZ in RWPE-1 and PCa cell lines was determined by western blot analysis, PrLZ was remarkably upregulated in PCa cells. ${ }^{* * *} \mathrm{P}<0.001$, unpaired two-tailed Student's t-test. (E) PrLZ expression was detected by western blot analysis in LNcap and PC-3 cells after miR-449a overexpression lentivirus or NC lentivirus infected, miR-449a significantly suppressed the expression of PrLZ in LNcap and PC-3 cells. ${ }^{* *} \mathrm{P}<0.01,{ }^{* * *} \mathrm{P}<0.001$, unpaired two-tailed Student's t-test. (F) The luciferase activity decreased after miR-449a mimic transfection in PrLZ-3'-UTR-wt transfected PC-3 cells. However, miR-449a mimic was unavailable in PrLZ-3'-UTR-mu transfected PC-3 cells. ${ }^{* *}$ P $<0.01$, unpaired two-tailed Student's t-test. 
A

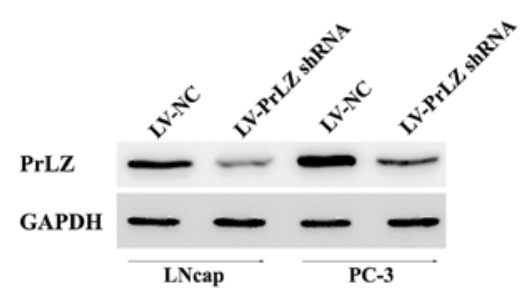

B

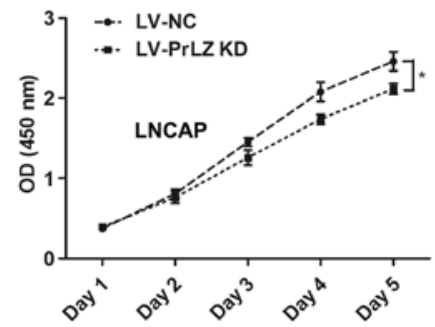

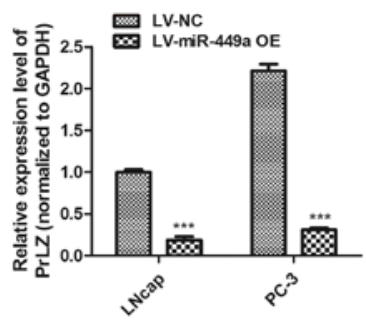

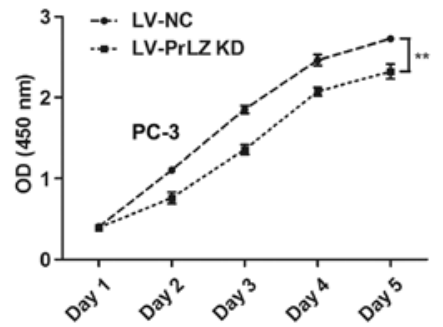

C
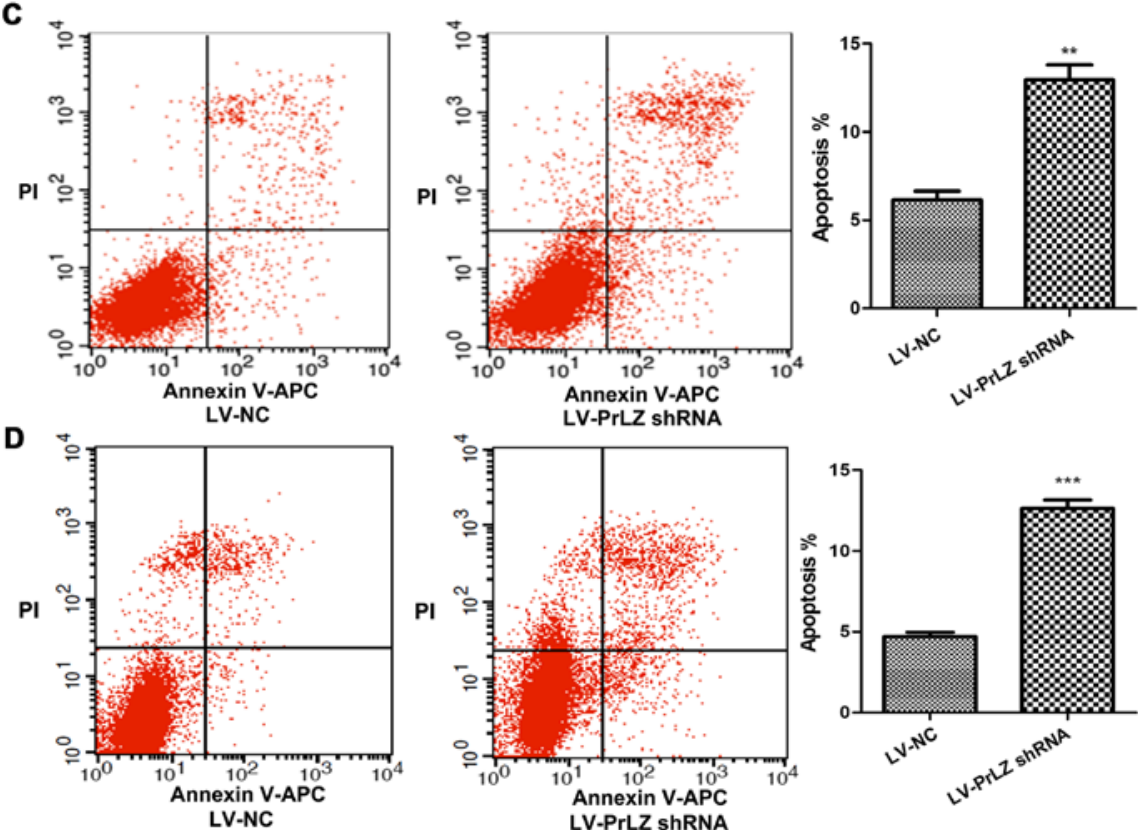

Figure 5. PrLZ-shRNA inhibits proliferation and promotes apoptosis in LNcap and PC-3 cells. (A) PrLZ was detected by western blotting in LNcap and PC-3 cells after PrLZ shRNA lentivirus or NC lentivirus infection. ${ }^{* * *} \mathrm{P}<0.001$, unpaired two-tailed Student's t-test. (B) PrLZ shRNA suppressed LNcap and PC-3 proliferation. ${ }^{*} \mathrm{P}<0.05,{ }^{* *} \mathrm{P}<0.01$, one-way ANOVA. (C and D) Downregulation of PrLZ promoted LNcap and PC-3 cell apoptosis. ${ }^{* *} \mathrm{P}<0.01,{ }^{* * * *} \mathrm{P}<0.001$, unpaired two-tailed Student's t-test.

apoptosis in LNcap and PC-3 cells (Fig. 2B). Moreover, we also assessed the impact of miR-449a on metastasis of PCa, we found that miR-449a obviously restricted invasion and tube formation ability in LNcap and PC-3 (Fig. 3). These results indicate that miR-449a is related to malignance of prostate cancer.

miR-449a targets PrLZ expression in PCa cells. To identify potential target of miR449a, we used a combination of three algorithms, TargetScan, miRDB and miRanda. Among these candidates, PrLZ was identified by all the three programs as potential target of miR-449a with highest predictive score (Fig. 4A). To validate our prediction, we detected PrLZ expression in corresponding 38 PCa specimens by qRT-PCR. Pearson's correlation analysis showed that the PrLZ expression negative correlated with miR-449a (Fig. 4B). IHC was performed to assess PrLZ expression in $\mathrm{PCa}$ and corresponding adjacent tissues, we found that PrLZ was upregulated in $\mathrm{PCa}$ tissues compared to adjacent specimens (Fig. 4C). Next, we further confirmed the target relationship of miR-449a/PrLZ via western blotting (Fig. 4D and E) and Luciferase assay (Fig. 4F). Our results indicate that PrLZ is specifically targeted by miR-449a in PCa cells.

Knockdown of PrLZ inhibits PCa progression. In order to confirm that miR-449a is a vital cancer suppressor via regulating PrLZ expression, we specially further investigated the function of PrLZ in PCa. PrLZ shRNA lentivirus (LV-PrLZ shRNA) was used to downregulate PrLZ expression, western blotting showed remarkable decrease of PrLZ in LNcap and PC-3 cells after PrLZ shRNA lentivirus infection (Fig. 5A). We found that PrLZ knockdown delayed LNcap and PC-3 cell proliferation (Fig. 5B). In addition, flow cytometry assay revealed remarkable elevation of apoptosis rate in LNcap and PC-3 cells after LV-PrLZ shRNA infection (Fig. 5C). Similar to miR-449a, PrLZ shRNA obviously restricted invasion and 

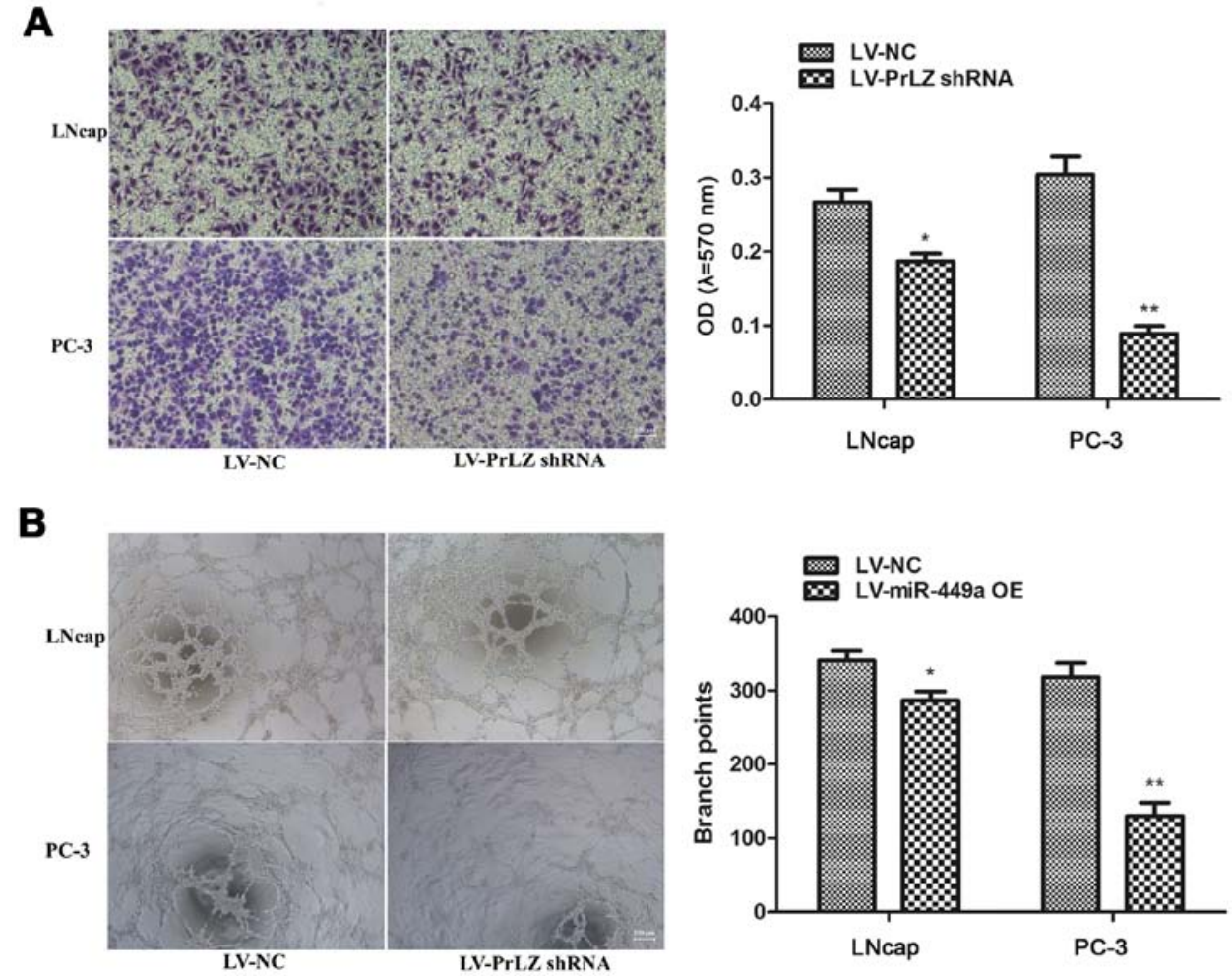

Figure 6. PrLZ-shRNA suppresses invasion and angiogenesis ability in LNcap and PC-3 cells. (A) PrLZ shRNA suppressed LNcap and PC-3 invasion. ${ }^{*}<0.05$, ${ }^{* *} \mathrm{P}<0.01$, unpaired two-tailed Student's t-test. (B) PrLZ shNRA inhibited LNcap and PC-3 induced huvec cells tube formation. * $\mathrm{P}<0.05$, ${ }^{* *} \mathrm{P}<0.01$, unpaired two-tailed Student's t-test.

Table II. Statistics of metastasis in vivo.

\begin{tabular}{lcccc}
\hline & & \multicolumn{3}{c}{ No. of metastasis } \\
\cline { 3 - 5 } Groups & $\begin{array}{c}\text { Total no. } \\
\text { of animals }\end{array}$ & $\begin{array}{c}\text { Liver } \\
\text { metastasis }\end{array}$ & $\begin{array}{c}\text { Lung } \\
\text { metastasis }\end{array}$ & Total \\
\hline LV-NC & 13 & 3 & 9 & 9 \\
LV-PrLZ shRNA & 13 & 1 & 3 & 4 \\
LV-miR-449a OE & 15 & 0 & 2 & 2 \\
\hline
\end{tabular}

tube formation ability in LNcap and PC-3 cells (Fig. 6). Our results prove that knockdown of PrLZ inhibits PCa progression.

miR-449a/PrLZ axis regulates $P C$ a cell invasion via adjusting stemness. Cancer stem cell is crucial in cancer progression and evolution, and the maintenance or resumption of stemness features are approved to be critical factors for PCa metastasis (17). We investigated the roles of miR-449a and PrLZ in regulating stemness features of $\mathrm{PCa}$ cells. E-cadherin, N-cadherin, vimentin, CD44, MMP2, MMP9 and Runx2 were detected as stemness or metastasis markers by western blotting (Fig. 7A). We found that E-cadherin was upregulated in LNcap and PC3 after LV-miR-449a OE or LV-PrLZ shRNA infection. Oppositely, N-cadherin, vimentin, CD44, MMP2, MMP9 and Runx2 were remarkably downregulated. Quantitative analysis results are shown (Fig. 7B-H). These results indicate that miR449a inhibits stemness features in LNcap and PC3 cells via targeting PrLZ, and miR449a/PrLZ axis may regulate $\mathrm{PCa}$ cell invasion via adjusting stemness.

miR-449a/PrLZ axis regulates PC-3 cell tumorigenesis and metastasis in vivo. PC-3 subcutaneously transplanted nude mice were used to clarify the function of miR-449a/PrLZ signal axis in vivo. We found that LV-miR-449a OE virus infection suppressed tumor formation in vivo. Similarly, PrLZ shRNA inhibited PC-3 cell tumorigenesis (Fig. 8A and B). We also observed tumor metastases in vivo, miR-449a and PrLZ shRNA inhibited the number of animals with tumor metastases, and data are shown in Table II. We further examined PrLZ and N-cadherin expression in tumor tissues obtained from nude mice. IHC showed that PrLZ and $\mathrm{N}$-cadherin were decreased in subcutaneous tumors (Fig. 8C and D). These results indicate that $\mathrm{miR}-449 \mathrm{a} / \mathrm{PrLZ}$ axis regulates $\mathrm{PCa}$ tumorigenesis and metastasis in vivo.

\section{Discussion}

As the deadly complication of malignant tumors, distant metastasis usually occurs in advanced stage of tumors such as prostate and lung cancer. The most common site of $\mathrm{PCa}$ metastasis is bone. Bone metastasis causes consequences, such as bone pain. Bone fractures and nerve compression, seriously affect the patient's quality of life $(18,19)$. Thus, it is urgent to develop accurate predictors and illuminate the mechanisms of PCa distant metastasis. Increasing research has indicated microRNAs play critical roles in tumor progression, and there is growing evidence that microRNAs are helpful tools in prostate cancer diagnosis and prognosis, but rarely has microRNA 
A

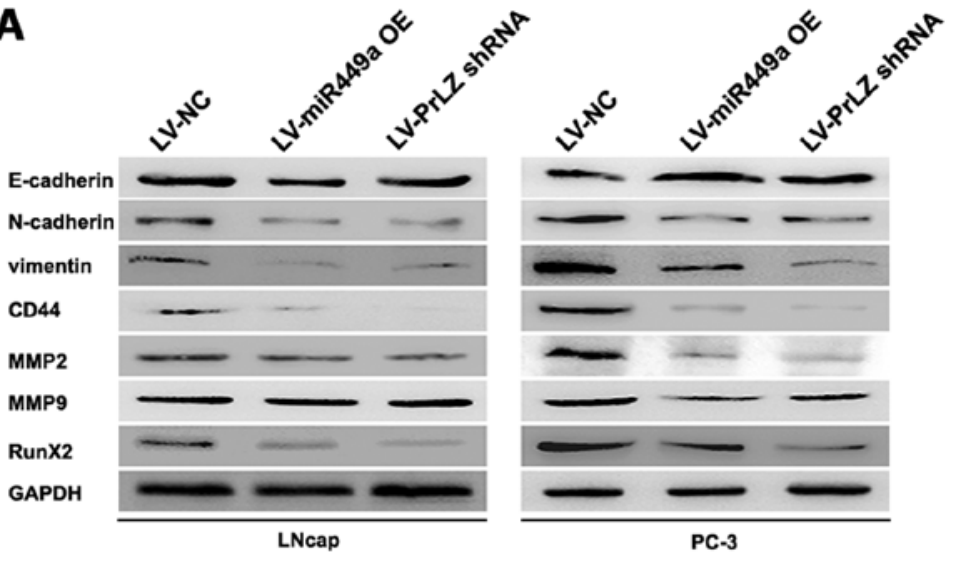

B

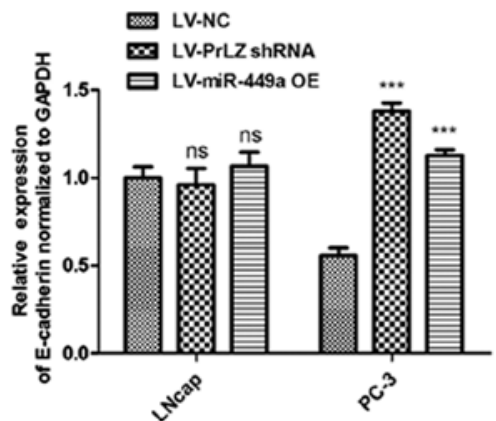

C
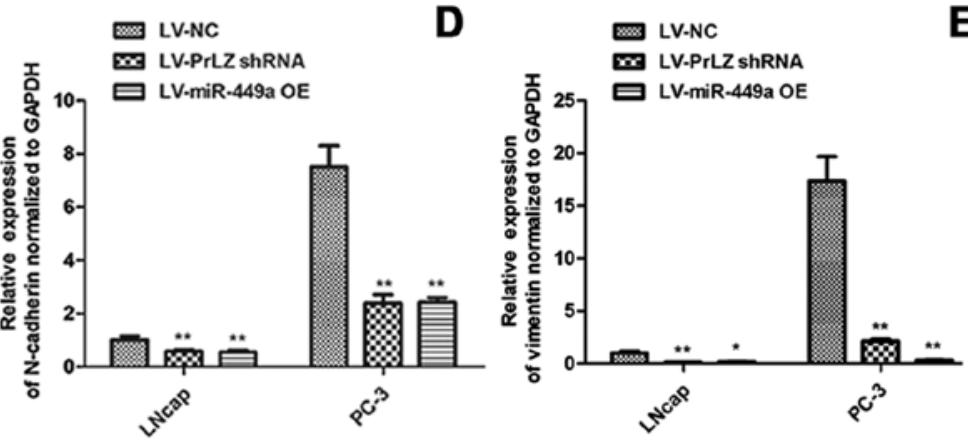

$\mathbf{E}$

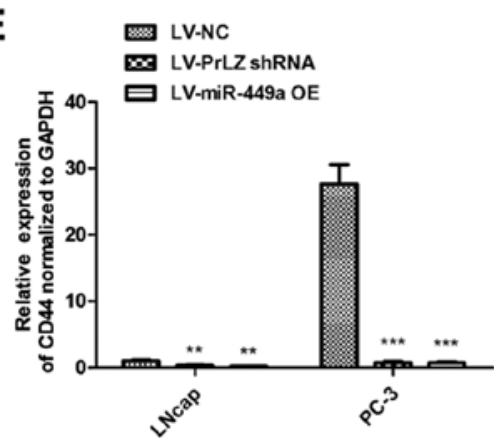

$\mathbf{F}$

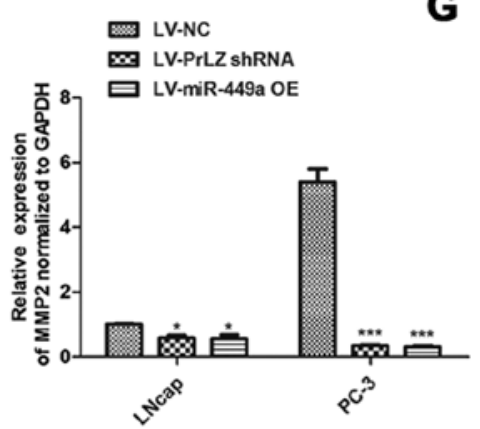

G

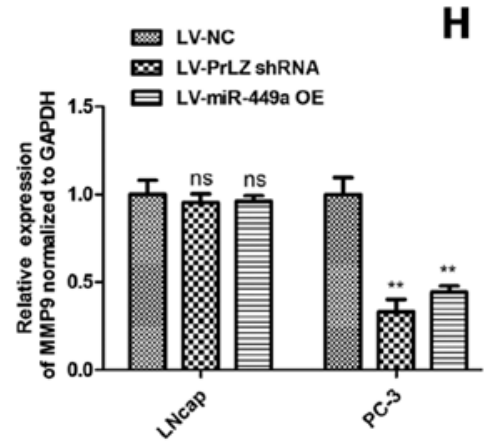

H

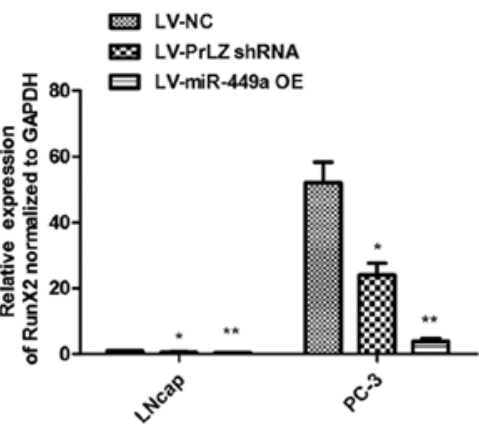

Figure 7. miR-449a and PrLZ shRNA regulate stemness and metastasis-related gene expression in LNcap and PC-3. (A) Western blot analysis of stemness related genes (E-cadherin, E-cadherin, vimentin and CD44) and MMP2, MMP9, RunX2 expression in LNcap and PC-3 cell after miR-449a overexpression lentivirus, PrLZ shRNA lentivirus or NC lentivirus infection. (B-H) Densitometric quantification of these above genes. ${ }^{*} \mathrm{P}<0.05,{ }^{* * *} \mathrm{P}<0.01,{ }^{* * *} \mathrm{P}<0.001$, unpaired two-tailed Student's t-test.

has been verified to be appropriate predictor of prostate cancer distant metastasis (20-22).

Previous studies reported that miR-449a was downregulated in various malignant tumors, including liver (23), non-small cell lung (24), gastric (25) and prostate cancer (26), and Mao et al (27) found that miR-449a enhances radiosensitivity by downregulation of c-Myc in prostate cancer cells. Thus, we wondered whether miR-449a was associated with prostate cancer progression and distant metastasis. In this study, we demonstrated that miR-449a was downregulated in PCa tissues, moreover, the expression of miR-449a was significantly lower in advanced stage of PCa specimens and the primary lesion tissues of PCa patients with postoperative bone metastasis. These findings indicated that miR-449a might be related to PCa clinical progression and distant metastasis. CCK-8 and apoptosis assay confirmed that the loss of miR-449a promoted PCa cells survival and proliferation.
As the most important characteristics of cancer cells, invasion and angiogenesis are the necessary abilities for tumor distal metastasis (28-31). Our results also showed that miR-449a suppressed invasion and angiogenesis of LNcap and PC-3 cells, which, respectively, represented androgen-dependent and androgen-independent PCa. These above results proved our conjecture that the loss of miR-449a was an important causality of PCa progression and distant metastasis. Research has suggested that microRNA exerted physiological function via negative regulating target genes. Studies reported that miR-449a targeted several genes in various cancers, including c-MET and Fos (23), c-Myc (27) and SOX4 (32). In this study, we further identified PrLZ as a functional target of miR-449a. As a novel prostate-specific and androgen-responsive gene, PrLZ was proved to contribute to malignant progression in prostate cancer $(13-15,33)$. However, there is still no evidence that PrLZ is associated with prostate cancer metastasis. Here 

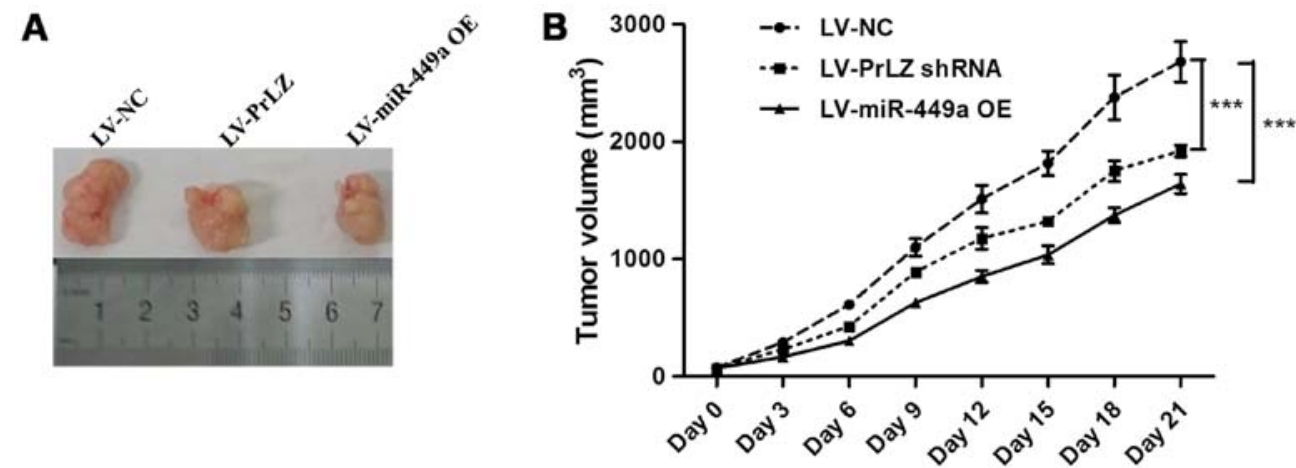

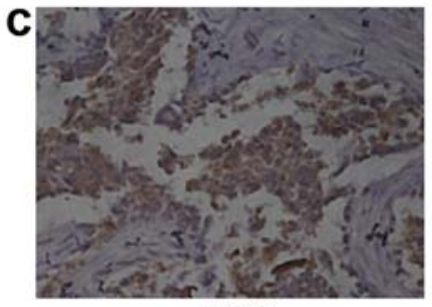

LV-NC

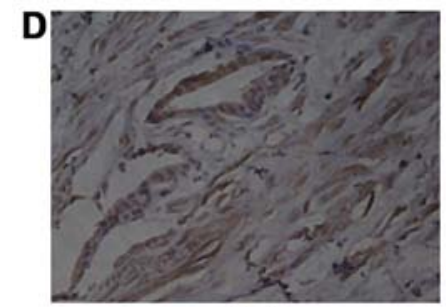

LV-NC

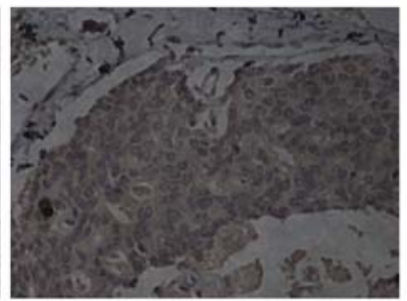

LV-PrLZ ShRNA

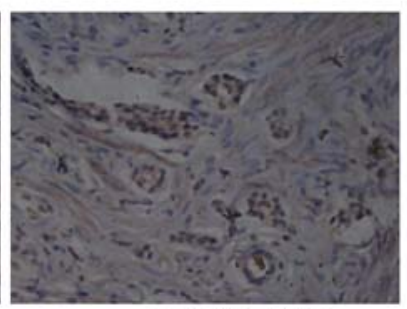

LV-PrLZ ShRNA

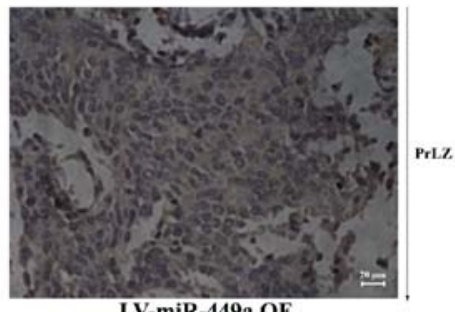

LV-miR-449a OE

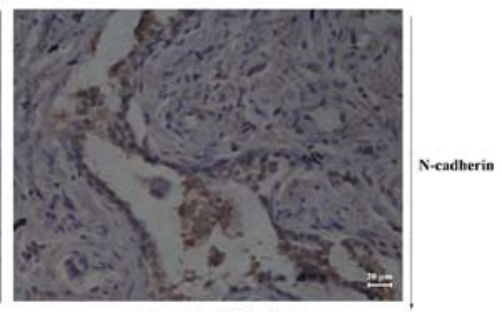

LV-miR-449a OE

Figure 8. miR-449a and PrLZ shRNA suppress tumor growth and inhibit stemness features of PCa in vivo. (A) Representative images of tumors isolated from nude mice. (B) Growth curves of tumors in nude mice. LV-miR-449a OE and LV-PrLZ shRNA lentivirus injection significantly delayed tumor growth. ${ }^{* * * *} \mathrm{P}<0.001$, one-way ANOVA. (C and D) IHC analysis of PrLZ and N-cadherin. miR-449a and PrLZ shRNA significantly inhibited N-cadherin expression in subcutaneous tumors.

we also further confirmed that PrLZ promoted PCa cell proliferation. Importantly, we further found that PrLZ shRNA remarkably supressed invasion and angiogenesis in LNcap and PC-3 cells. These above results prompted that miR-449a regulated $\mathrm{PCa}$ progression and metastasis via targeting PrLZ. The decrease of miR-449a caused increase of PrLZ in primary lesions and is critical in PCa progression and distal metastasis, in vivo studies further confirmed our conclusions. However, how did miR-449a/PrLZ axis regulated prostate cancer distant metastasis? Chen et al (23) revealed that miR449a inhibited the epithelial-mesenchymal transition (EMT) of hepatocellular carcinoma, and as reported in previous studies, stemness and EMT of cancer cells played critical roles in cancer metastasis (19,34-36), thus, we wondered whether miR-449a/PrLZ axis was associated with EMT of prostate cancer cells. We found that miR-449a and PrLZ shRNA significantly suppressed $\mathrm{N}$-cadherin, vimentin and CD44 expression in PCa cells. IHC assay also displayed that $\mathrm{N}$-cadherin decreased in tumors resected from PC-3 xenograft models, in which PrLZ was downregulated by miR449a or PrLZ shRNA. Our results indicated that miR-449a/PrLZ axis might regulate prostate cancer invasion through EMT and stemness maintenance.

In conclusion, in the present study, we confirmed that the loss of miR-449a caused PrLZ overexpression promoting prostate cancer progression and distant metastasis, the clinical diagnotic value of miR-449a and PrLZ need further study.

\section{Acknowledgements}

We wish to acknowledge the patients enrolled in the present study for their participation, and the Department of Urology, The First Affiliated Hospital of Wenzhou Medical University, for its collaboration in providing the human specimens and clinical information used in this study. We also particularly acknowledge Shanghai R\&S Biotechnology Co., Ltd., for its technical assistance and providing lentivirus used in this study. The study was also supported by grants from the National Natural Science Funds of China (no. 81301791) and the Foundation for Outstanding Young Scientist of Shandong Province (no. BS2013YY030).

\section{References}

1. Adisa JO, Egbujo EC, Ibrahim B, Musa B and Madukwe J: Expression of some selected cytokeratins and Ki67 protein in prostatic tumor: Can these be used as tumor markers. Pan Afr Med J 20: 46, 2015.

2. Miller KD, Siegel RL, Lin CC, Mariotto AB, Kramer JL, Rowland JH, Stein KD, Alteri R and Jemal A: Cancer treatment and survivorship statistics, 2016. CA Cancer J Clin 66: 271-289, 2016. 
3. Qi JL, Wang LJ, Zhou MG, Liu YN, Liu JM, Liu SW, Zeng XY and Yin P: Disease burden of prostate cancer among men in China, from 1990 to 2013. Zhonghua Liu Xing Bing Xue Za Zhi 37: 778-782, 2016 (In Chinese).

4. Pound CR, Partin AW, Eisenberger MA, Chan DW, Pearson JD and Walsh PC: Natural history of progression after PSA elevation following radical prostatectomy. JAMA 281: 1591-1597, 1999.

5. Logothetis CJ and Lin SH: Osteoblasts in prostate cancer metastasis to bone. Nat Rev Cancer 5: 21-28, 2005

6. Kosuda S, Yoshimura I, Aizawa T, Koizumi K, Akakura K, Kuyama J, Ichihara K, Yonese J, Koizumi M, Nakashima J, et al: Can initial prostate specific antigen determinations eliminate the need for bone scans in patients with newly diagnosed prostate carcinoma? A multicenter retrospective study in Japan. Cancer 94: 964-972, 2002.

7. Tanaka N, Fujimoto K, Shinkai T, Nakai Y, Kuwada M, Anai S, Miyake M, Hirayama A, Hasegawa M and Hirao Y: Bone scan can be spared in asymptomatic prostate cancer patients with PSA of $<=20 \mathrm{ng} / \mathrm{ml}$ and Gleason score of $<=6$ at the initial stage of diagnosis. Jpn J Clin Oncol 41: 1209-1213, 2011.

8. Lilleby W and Fosså SD: Chemotherapy in metastatic renal cell cancer. World J Urol 23: 175-179, 2005

9. Todorova K, Metodiev MV, Metodieva G, Zasheva D, Mincheff M and Hayrabedyan S: miR-204 is dysregulated in metastatic prostate cancer in vitro. Mol Carcinog 55: 131-147, 2016.

10. Siu MK, Tsai YC, Chang YS, Yin JJ, Suau F, Chen WY and Liu YN: Transforming growth factor- $\beta$ promotes prostate bone metastasis through induction of microRNA-96 and activation of the mTOR pathway. Oncogene 34: 4767-4776, 2015.

11. Browne G, Taipaleenmäki H, Stein GS, Stein JL and Lian JB: MicroRNAs in the control of metastatic bone disease. Trends Endocrinol Metab 25: 320-327, 2014.

12. Abba M, Patil N, Leupold JH and Allgayer H: MicroRNAs - from metastasis prediction to metastasis prevention? Mol Cell Oncol 3 : e1074336, 2015.

13. Wang R, Xu J, Mabjeesh N, Zhu G, Zhou J, Amin M, He D, Marshall FF, Zhau HE and Chung LW: PrLZ is expressed in normal prostate development and in human prostate cancer progression. Clin Cancer Res 13: 6040-6048, 2007.

14. Li L, Zhang D, Zhang L, Zhu G, Sun Y, Wu K, Wang X and He D: PrLZ expression is associated with the progression of prostate cancer LNCaP cells. Mol Carcinog 48: 432-440, 2009.

15. Li L, Xie H, Liang L, Gao Y, Zhang D, Fang L, Lee SO, Luo J, Chen X, Wang X, et al: Increased PrLZ-mediated androgen receptor transactivation promotes prostate cancer growth at castration-resistant stage. Carcinogenesis 34: 257-267, 2013.

16. Upile T, Jerjes W, Radhi H, Al-Khawalde M, Kafas P, Nouraei S and Sudhoff H: Vascular mimicry in cultured head and neck tumour cell lines. Head Neck Oncol 3: 55, 2011.

17. Deep G, Jain AK, Ramteke A, Ting H, Vijendra KC, Gangar SC, Agarwal C and Agarwal R: SNAI1 is critical for the aggressiveness of prostate cancer cells with low E-cadherin. Mol Cancer 13: 37, 2014.

18. Keller ET, Dai J, Escara-Wilke J, Hall CL, Ignatoski K, Taichman RS and Keller J: New trends in the treatment of bone metastasis. J Cell Biochem 102: 1095-1102, 2007.

19. Weidle UH, Birzele F, Kollmorgen G and Rüger R: Molecular mechanisms of bone metastasis. Cancer Genomics Proteomics 13: $1-12,2016$
20. Singh R, Ramasubramanian B, Kanji S, Chakraborty AR, Haque SJ and Chakravarti A: Circulating microRNAs in cancer: Hope or hype? Cancer Lett 381: 113-121, 2016.

21. White NM, Fatoohi E, Metias M, Jung K, Stephan C and Yousef GM: Metastamirs: A stepping stone towards improved cancer management. Nat Rev Clin Oncol 8: 75-84, 2011.

22. Ell B and Kang Y: MicroRNAs as regulators of bone homeostasis and bone metastasis. Bonekey Rep 3: 549, 2014.

23. Chen SP, Liu BX, Xu J, Pei XF, Liao YJ, Yuan F and Zheng F: MiR-449a suppresses the epithelial-mesenchymal transition and metastasis of hepatocellular carcinoma by multiple targets. BMC Cancer 15: 706, 2015.

24. Luo W, Huang B, Li Z, Li H, Sun L, Zhang Q, Qiu X and Wang E: MicroRNA-449a is downregulated in non-small cell lung cancer and inhibits migration and invasion by targeting c-Met. PLoS One 8: e64759, 2013.

25. Li Q, Peng J, Li X, Leng A and Liu T: miR-449a targets Flot2 and inhibits gastric cancer invasion by inhibiting TGF- $\beta$-mediated EMT. Diagn Pathol 10: 202, 2015.

26. Noonan EJ, Place RF, Pookot D, Basak S, Whitson JM, Hirata H, Giardina C and Dahiya R: miR-449a targets HDAC-1 and induces growth arrest in prostate cancer. Oncogene 28: 1714-1724, 2009.

27. Mao A, Zhao Q, Zhou X, Sun C, Si J,Zhou R, Gan L and Zhang H: MicroRNA-449a enhances radiosensitivity by downregulation of c-Myc in prostate cancer cells. Sci Rep 6: 27346, 2016.

28. Graff JR and Zimmer SG: Translational control and metastatic progression: Enhanced activity of the mRNA cap-binding protein eIF-4E selectively enhances translation of metastasisrelated mRNAs. Clin Exp Metastasis 20: 265-273, 2003.

29. Kelly T, Suva LJ, Huang Y, Macleod V, Miao HQ, Walker RC and Sanderson RD: Expression of heparanase by primary breast tumors promotes bone resorption in the absence of detectable bone metastases. Cancer Res 65: 5778-5784, 2005.

30. Miyamoto N, Yamamoto H, Taniguchi H, Miyamoto C, Oki M, Adachi Y, Imai K and Shinomura Y: Differential expression of angiogenesis-related genes in human gastric cancers with and those without high-frequency microsatellite instability. Cancer Lett 254: 42-53, 2007.

31. Perlikos F, Harrington KJ and Syrigos KN: Key molecular mechanisms in lung cancer invasion and metastasis: A comprehensive review. Crit Rev Oncol Hematol 87: 1-11, 2013.

32. Sandbothe M, Buurman R, Reich N, Greiwe L, Vajen B, Gürlevik E, Schäffer V, Eilers M, Kühnel F, Vaquero A, et al: The microRNA-449 family inhibits TGF- $\beta$-mediated liver cancer cell migration by targeting SOX4. J Hepatol 66: 1012-1021, 2017.

33. Zhang H, Wang J, Pang B, Liang RX, Li S, Huang PT, Wang R, Chung LW, Zhau HE, Huang C, et al: PC-1/PrLZ contributes to malignant progression in prostate cancer. Cancer Res 67: 8906-8913, 2007.

34. Nakazawa M and Kyprianou N: Epithelial-mesenchymaltransition regulators in prostate cancer: Androgens and beyond. J Steroid Biochem Mol Biol 166: 84-90, 2017.

35. Diepenbruck M and Christofori G: Epithelial-mesenchymal transition (EMT) and metastasis: Yes, no, maybe? Curr Opin Cell Biol 43: 7-13, 2016.

36. Kim EK, Choi EJ and Debnath T: Role of phytochemicals in the inhibition of epithelial-mesenchymal transition in cancer metastasis. Food Funct 7: 3677-3685, 2016. 